\title{
Editorial
}

\section{Engineering Applications of Intelligent Monitoring and Control 2014}

\author{
Qingsong Xu, ${ }^{1}$ Pak-Kin Wong, ${ }^{1}$ Minping Jia, ${ }^{2}$ Chengjin Zhang, ${ }^{3}$ and Ping-Lang Yen \\ ${ }^{1}$ Department of Electromechanical Engineering, Faculty of Science and Technology, University of Macau, \\ Avenida da Universidade, Taipa, Macau \\ ${ }^{2}$ School of Mechanical Engineering, Southeast University, Nanjing, Jiangsu 211189, China \\ ${ }^{3}$ School of Control Science and Engineering, Shandong University, Jinan, Shandong 250061, China \\ ${ }^{4}$ Department of Bio-Industrial Mechatronics Engineering, National Taiwan University, No. 1 Section 4 Roosevelt Road, \\ Taipei 10617, Taiwan \\ Correspondence should be addressed to Qingsong Xu; qsxu@umac.mo
}

Received 21 December 2014; Accepted 21 December 2014

Copyright (C) 2015 Qingsong Xu et al. This is an open access article distributed under the Creative Commons Attribution License, which permits unrestricted use, distribution, and reproduction in any medium, provided the original work is properly cited.

Intelligent monitoring and control is an important issue in various engineering domains including mechanical engineering, electrical engineering, control engineering, civil engineering, biomedical engineering, and micro/nanoengineering. Unlike traditional method, intelligent approaches enable some attractive advantages to the systems, namely, perception, reasoning, learning, and making decisions from incomplete information. Such merits are essential for lots of engineering applications without human interaction. Following the inaugural special issue, the main focus of this year's annual issue is on emerging intelligent system approaches with the goal of monitoring and controlling various physical processes with no or very little human interaction. The objective of this issue is to summarize the recent emerging developments and ideas in concerned domains of intelligent monitoring and control.

After a thorough review process, 35 out of a total of 82 submitted papers have been selected in this annual issue, which fall into three main groups.

(I) Intelligent Monitoring, Detection, and Identification. As a novel energy source, microwave heating mode has been applied widely in many industrial fields. However, information about temperature distribution is complex but of critical importance for the control of various microwave applications. $\mathrm{X}$. Shen et al.'s “Temperature Distribution Monitoring Using Ultrasonic Thermometry Based on Markov Radial Basis
Function Approximation and Singular Values Decomposition" devises an innovative way of temperature distribution monitoring using ultrasonic thermometry in microwave field. Simulation experiments under different conditions are carried out to investigate the performance of the reported methodology.

Externally prestressed concrete structure is broadly applied in highway bridges, urban bridges, and railway bridges with the development of external prestress technology. To effectively control the operating state and the bearing capacity of bridges, it is important to identify the prestress force of externally prestressed concrete bridge. Motivated by this, a prestress force identification method for externally prestressed concrete uniform beam based on the frequency equation and the measured frequencies is developed in L. Shi et al.s "Prestress Force Identification for Externally Prestressed Concrete Beam Based on Frequency Equation and Measured Frequencies". The effectiveness of the prestress force identification method is demonstrated by the singlespan externally prestressed concrete beam and two-span externally prestressed concrete beam tests.

Video-based traffic surveillance systems are widely used in traffic monitoring and management. Lane detection is a crucial process in video-based transportation monitoring system. J. Ren et al's "Lane Detection in Video-Based Intelligent Transportation Monitoring via Fast Extracting and Clustering of Vehicle Motion Trajectories" proposes a novel 
method to detect the lane center via rapid extraction and high accuracy clustering of vehicle motion trajectories. Experimental results prove that the proposed method can detect lane center position efficiently, the affected time of subsequent tasks can be reduced obviously, and the safety of traffic surveillance systems can be enhanced significantly.

Unmanned aerial vehicles (UAVs) have been applied in a wide range of scenarios. With shared wireless communication media, they are highly sensitive to denial of service attacks, and jamming is one of these attacks. Y. Zhang and L. Yang's "Triangle and GA Methods for UAVs Jamming" focuses on how to jam UAVs network efficiently. Results show that the Triangle and genetic algorithm (GA) methods perform better than Random method, and some remarkable conclusions are drawn.

Traditional video sources mostly adopt interlaced scanning mode. In the process of converting interlaced scanning to progressive scanning, interline flickers, saw-tooth, and creeping could be found in motion images. Based on lowangle detection and multiangle extraction, an interfield and intrafield weighted interpolative algorithm is proposed in J. Qiang et al.s “An Interfield and Intrafield Weighted Interpolative Deinterlacing Algorithm Based on Low-Angle Detection and Multiangle Extraction". Reported experimental results show that the proposed algorithm could improve peak signalto-noise ratio (PSNR), restrain some interlace phenomenon, and reach better visual effects.

Medicine has received noteworthy contributions, which have allowed for great advancement in medical activity within various scenarios. The acoustic analysis is a complementary technique to methods based on the direct inspection of vocal folds. A. I. R. Fontes et al.'s "Classification System of Pathological Voices Using Correntropy" introduces a new low-complexity approach for the detection and classification of laryngeal pathologies. This system uses a measure of information theory known as correntropy to characterize vocal pathologies by means of spectral characteristics and statistical moments of high order involving the vocal signal. The system features high success rates and low computational complexity associated with a simple classification stage based on Euclidian distance.

Various crustal movements and anthropogenic activities can result in ground deformation. However, accurate detection of small deformation over large areas was very difficult in the past. B. Hu's "Monitoring of Ground Deformation due to Excessive Withdrawal of Natural Gas Using SBAS" investigates the ground deformation in Chiba Prefecture, Japan, using the Small Baseline Subset (SBAS) technique. The obtained result has significance to the precaution of gas mining geologic hazard and environment disruption.

In structural health monitoring (SHM), various sensors are installed at critical locations of a structure. An objective comparison of the sensor data at different time ranges is essential for assessing the structural condition or excessive load experienced by the structure which leads to potential damage in the structure. To estimate the reliability of the data and potential damage using the statistical pattern matching techniques, a relationship between the data from various sensors is established by M. S. Islam and A. Bagchi's "Statistical
Pattern-Based Assessment of Structural Health Monitoring Data". The proposed methodologies have been developed and validated using sensor data obtained from an instrumented bridge and road test data from heavy vehicles. Results demonstrate that it has high potential in assessing structural conditions, especially when the data are noisy and susceptible to environmental disturbances.

Rotor resistance identification is one of the most critical factors affecting the theoretical study and applications of AC motor's control for high performance variable frequency speed adjustment. B. Fan et al.s "Rotor Resistance Online Identification of Vector Controlled Induction Motor Based on Neural Network" proposes a novel model for rotor resistance parameters identification based on Elman neural networks. Simulation and experimental results show that the proposed method owns extensive adaptability and performs very well in application to vector controlled induction motor. This identification method is able to enhance the performance of induction motor's variable-frequency speed regulation.

Cloud based large-scale online services are faced with regionally distributed stochastic demands for various resources. With multiple regional cloud data centers, a crucial problem that needs to be settled is how to properly place resources to satisfy massive stochastic demands from many different regions. W. Wei et al.s "A Time-Efficient Solution to the General Resource Placement Problem in Cloud" proposes an efficient algorithm, named discrete function based unbound resource placement (D-URP). Experiments show that DURP can averagely achieve at least $97 \%$ revenue of optimal solution, with reducing time by three orders of magnitude.

Grain moisture content is one of the key indexes of grain storage security, and it is an important control index in the process of grain processing. The online measurement of grain moisture has been a problem of the realization of automatic operation of grain dryers. Z. Liu et al.s "Research on Online Moisture Detector in Grain Drying Process based on V/F Conversion" proposes an online resistance grain moisture detector, which is based on the model of the relationship between measurement frequency and grain moisture, and the nonlinear correction method of temperature. The performance of the online moisture detector is tested in a self-designed experimental system.

Mixed-model assembly line has been widely used in various industries, including automotive assembly, mobile phone making, and computer production. Z. Yang et al.'s "Modeling of RFID-Enabled Real-time Manufacturing Execution System in Mixed-Model Assembly Lines" introduces RFID technology to manufacturing workshop to convert manufacturing objects to be "smart objects" which are enabled to interact dynamically in real-time. The proposed design scheme has important reference value for the applications of RFID in multiple manufacturing fields and also lays a vital research foundation to leverage digital and networked manufacturing system towards intelligence.

(II) Prediction, Prognosis, and Diagnosis. Performance degradation forecast technology for quantitatively assessing degradation states of aeroengine is one of the most important 
technologies aiming at improving the safety, reliability, and maintenance of aeroengine. Based on the grey system theory, N. Zhao et al.'s "A GM $(1,1)$ Markov Chain-Based Aeroengine Performance Degradation Forecast Approach Using Exhaust Gas Temperature" introduces the time series forecast to the forecast modeling of aeroengine gas path performance parameters. Specifically, a GM $(1,1)$ Markov chain-based approach is introduced to forecast exhaust gas temperature by taking the advantages of GM $(1,1)$ model in time series and the advantages of Markov chain model in dealing with highly nonlinear and stochastic data caused by uncertain factors. Comparison results show that the proposed model is capable of forecasting exhaust gas temperature accurately and it can effectively reflect the random fluctuation characteristics of exhaust gas temperature changes over time.

Accurate prediction models for air pollutants are crucial for forecast and health alarm to local inhabitants. The commonly used discrete wavelet transform (DWT) has a significant demand in model complexity, namely, the training time and the model size of the prediction model. C. M. Vong et al.'s "Variation-Oriented Data Filtering for Improvement in Model Complexity of Air Pollutant Prediction Model" reports on a new method called variation-oriented filtering (VF) to remove the data with low variation, which can be considered as noise to a prediction model. By selecting the SO2 (sulfur dioxide) level in Macau as a test case, experimental results demonstrate that the proposed new VF algorithm can effectively and efficiently reduce the model complexity with improvement in predictive accuracy.

Fatigue crack is one of the reasons for structural failures of infrastructures such as bridges and tunnels. Tomography is an emerging technique for nondestructive evaluation and aims to visualize the internal structure of various types of objects. Acoustic emission (AE) tomography, which is based on the time-travel tomography with $\mathrm{AE}$ events as its signal sources, is a relatively new visualization tool for inspecting and locating the internal damages in the structures. Y. Jiang et al.s "Simulation and Experimental Investigation on the AE Tomography to Improve AE Source Location in the Concrete Structure" applies the AE tomography to examine the damage in a typical heterogeneous concrete structure. Its effectiveness has been verified by carrying out experimental and numerical simulation studies.

Cloud application provides access to large pool of virtual machines for building high-quality applications to satisfy customers' requirements. A challenge lies in how to predict virtual machine response time because it determines when the dynamic scalable virtual machines could be adjusted. To address this critical issue, a prediction virtual machine response time method, which is based on genetic algorithmback propagation (GA-BP) neural network, is proposed by J. Guo et al.s "Research on Virtual Machine Response Time Prediction Method Based on GA-BP Neural Network". The effectiveness of the method has been confirmed through largescale experimental studies.

As is known, a crack may initiate under the alternative load, which will reduce the structural strength and may lead to tooth fracture with crack propagation. If the tooth crack can be detected early and crack propagation can be monitored, a disastrous breakdown can be avoided by replacing damaged gear. Considering the misalignment of gear root circle and base circle as well as accurate transition curve, an improved mesh stiffness model for healthy gear is proposed by H. Ma et al.'s "Fault Feature Analysis of a Cracked Gear Coupled Rotor System". The model has been validated through a comparison study with finite element method.

In modern rapidly developing industries, the rolling bearing, a vital component of most rotary machines, faces an increasingly complex working environment. Yet, the fault diagnosis precision for rolling bearings under variable conditions is usually unsatisfactory. To solve this problem, a fault diagnosis method combining Hilbert-Huang transform (HHT), singular value decomposition (SVD), and Elman neural network is proposed in H. Liu et al.s "Rolling Bearing Fault Diagnosis under Variable Conditions Using Hilbert-Huang Transform and Singular Value Decomposition". Experimental results show that the proposed method can effectively classify rolling bearing fault modes with high precision under different operating conditions.

When single phase to ground (SPG) fault occurs in power distribution network, fault line selection method is always study emphasis. For the arc suppression coil grounding system, the fault current is very weak, and judging the fault line accurately becomes more difficult. X. Wang et al.'s "A Novel Fault Line Selection Method Based on Improved Oscillator System of Power Distribution Network" proposes a novel method of fault line selection based on improved oscillator system (IOS) technology. Simulation results show that the proposed method can accurately judge fault line and healthy line in strong noisy background. Besides, the nondetection zones of the proposed method are elaborated.

Nowadays, green energy has gained increasing support among the people and electric energy has become much more popular. As the core cell of several types of electronic equipment and complex systems, Li-ion batteries are a critical electronic component, and the failure of $\mathrm{Li}$-ion batteries can lead to system malfunction, degraded systems, and even human casualties and financial losses. Y. Tian et al.s "Artificial Fish Swarm Algorithm-Based Particle Filter for Li-Ion Battery Life Prediction" presents an intelligent online prognostic approach for predicting the remaining useful life (RUL) of lithium-ion (Li-ion) batteries based on artificial fish swarm algorithm (AFSA) and particle filter (PF), which is an integrated approach combining model-based method with datadriven method.

The precision CNC coordinate boring machine is a tool for processing complex box-type components. Thermal error accounts for a larger proportion of total error as the machine tools become more sophisticated. However, the accuracy decreases and becomes far lower than the initial design value after the machine is used for a long period of time. To improve the CNC machine tools precision, a thermal error modeling for the motorized spindle is proposed in J. Yang et al.'s "Thermal-Induced Errors Prediction and Compensation for a Coordinate Boring Machine Based on Time Series Analysis". It is based on time series analysis in consideration of the length of cutting tools and thermal declined angles, and 
the real-time error compensation is implemented. Experimental results demonstrate the effectiveness of the proposed methodology of measurement, modeling, and compensation.

The emergence of complex machinery and equipment in some domains demands efficient fault diagnosis methods. M. Inacio et al.s "Fault Diagnosis with Evolving Fuzzy Classifier based on Clustering Algorithm and Drift Detection" reports the application of an evolving fuzzy classifier for fault diagnosis based on a new approach that combines a recursive clustering algorithm and a drift detection method. The classifier proposed in this work is evaluated in fault diagnosis experiments which are performed with a DC drive system model. Experiments show that the classifier is able to detect and classify all faults with a high performance, even in the presence of outliers and noise.

(III) Intelligent Sensing and Control. Based on the approach of intelligent self-adjustment, adaptive control is a powerful control scheme for dynamic system with high uncertainty. Yet, majority of existing adaptive neural control schemes are based on stochastic gradient-descent backpropagation (SGBP), which suffers from local minima problem. To overcome this issue, P. K. Wong et al.'s "Adaptive Control Using Fully Online Sequential-Extreme Learning Machine and a Case Study on Engine Air-Fuel Ratio Regulation" proposes an improved version of regularized online sequential-extreme learning machine (ReOS-ELM), entitled fully online sequential-extreme learning machine (FOS-ELM). To demonstrate its effectiveness, FOS-ELM is applied to the adaptive control of engine air-fuel ratio based on a simulated engine model. Result reveals that the reported FOS-ELM achieves better tracking and convergence performance than conventional approach. This is enabled by the essence of FOS-ELM method, which tends to learn the unknown engine model globally whereas SGBP tends to "forget" what it has learnt.

As an essential technique in computer vision, visual tracking has been applied in many fields such as vehicle tracking, medical imaging, robotics, and surveillance. In practice, challenges exist in the presence of occlusion, illumination changes, viewpoints variation, and noise interruption. L. He et al.'s "Multifeatures Based Compressive Sensing Tracking" casts tracking as a sparse approximation problem in a particle filter framework based on multifeatures to benefit from the development of compressive sensing. The reported experimental results demonstrate that the proposed tracker has better robustness as compared with the other four different algorithms.

Piezoelectric actuators (PEAs) have been widely used in nanopositioning systems due to their fast response and nanometer scale resolution. However, the existing hysteresis in PEAs can greatly limit system performance. G. Zhang et al's "Iterative Learning Control of Hysteresis in Piezoelectric Actuators" develops the convergence criteria of an iterative learning control on the whole desired trajectory to obtain the hysteresis-compensating feedforward input in hysteretic systems. Specifically, Prandtl-Ishlinskii model is used to capture the nonlinear behavior in piezoelectric actuators. Using the proposed control algorithm, experimental studies show that the tracking error of piezoelectric actuator has been significantly reduced to $0.15 \%$ of the total displacement, standing for a high level of tracking precision.

Reliable power and temperature control in pressurized water reactor (PWR) nuclear power plant is necessary to guarantee high efficiency and plant safety. Nuclear power plants are generally nonlinear, due to the frequent changes of the operating point right across the whole operation range. X. Liu and M. Wang's "Nonlinear Fuzzy Model Predictive Control for a PWR Nuclear Power Plant" proposes a nonlinear fuzzy model predictive control (MPC), by incorporating the realistic constraints, to realize the plant optimization. Simulation results demonstrate that the improved performance of the reported control is obtained as compared to traditional PID controller for a three-mile island- (TMI-) type PWR.

Wireless sensor network (WSN) has been widely used in many areas such as military, civilian use, and medical biology. In many mobile WSN applications, the low power problem is often ignored to maintain the real-time property of monitoring nodes. To solve the long-time working problems of mobile nodes and the whole WSN network, a new working method is developed by X. Du et al.s "A Real-Time and Low Power Working Method for Mobile WSN". It is demonstrated that the proposed method can effectively satisfy the requirements of real-time and low power consumption.

With the development of power system, the demands of the load are increasing. Power systems are more likely to encounter some problems such as oscillations and complex nonlinear phenomena due to the extension of power grid scale and some emergencies. Therefore, ways of maintaining the reliability and stability of power system are attracting extensive research interests. Z. Xiu-yu et al.'s “Adaptive Dynamic Surface Control for Generator Excitation Control System" proposes a novel neural network and tracking error transformed function based on adaptive dynamic surface control scheme for the generator excitation control system which is equipped with static var compensator (SVC). It is proved that the new scheme can make the system semiglobally uniformly ultimately bounded. Simulation results show the effectiveness of this control scheme.

Ball mill pulverizing system has advantages of good adaptability for mineral aggregate of different types, and it has been widely employed in power plant, cement industry, chemical industry, metallurgy, and many other fields. Exact measurement of fill level is a key and basic factor for realizing automatic, reliable, and efficient operation of the mill system. However, existing technique for collecting the vibration signal from mill shell is immature. To this end, a novel method for collecting vibration data from mill shell is proposed by $\mathrm{P}$. Huang et al.'s "Study on the Method for Collecting Vibration Signals from Mill Shell Based on Measuring the Fill Level of Ball Mill". Experimental result shows that vibration signals, which are collected by the proposed method, present a high sensitivity to the changes on fill level as compared with vibration data of mill bearing, which provides a reliable basis for accurate measurement of the fill level.

For the operation of the supercritical once-through boiler generation units, the control of the temperature at intermediate point (IPT) is highly significant. The commonly used 
PID control cannot achieve the optimal performances as the units' dynamic characteristic changes at different working points due to the severe nonlinearity. To address the problem, a new control algorithm using affine nonlinear system is reported by $\mathrm{H}$. Zhou et al.s “Temperature Control via Affine Nonlinear Systems for Intermediate Point of Supercritical Once-Through Boiler Units". Comparative study shows that the new method has a better performance with a quicker response time and a smaller overshoot, which demonstrates the potential improvement for the supercritical once-through boiler generation unit control.

Continuously variable transmission (CVT) is a type of automatic transmission that has been increasingly used in automotive applications. Yet, it is only applicable to low-power passenger cars because of its low torque capacity. To overcome this issue, dual-belt Van Doorne's CVT (DBVCVT) system has been previously proposed, which is applicable to heavyduty vehicles. To extend the research, $\mathrm{Z}$. Xie et al.'s "Intelligent Multiobjective Slip and Speed Ratio Control of a Novel DualBelt Continuously Variable Transmission for Automobiles" proposes an intelligent multiobjective fuzzy controller for slip and speed ratio control of DBVCVT. The controller aims to safely control the clamping forces of both the primary and the secondary pulleys in order to improve the transmission efficiency, achieve the accurate speed ratio, and avoid the belt slip under different engine loads and vehicle speeds. Experimental results show that the proposed controller for slip and speed ratio regulation of DBVCVT is effective and performs well under different user-defined weights.

Rehabilitation robots (RRs) are becoming increasingly common in upper extremity rehabilitation. However, most of the existing RRs suffer from a number of challenges. Aiming at aiding a person to achieve a desired functional task (e.g., tracking trajectory) based on assisted-as-needed principle, a new adaptive inverse optimal hybrid control combining inverse optimal control and actor-critic learning is proposed by F. Meng and Y. Dai's "Adaptive Inverse Optimal Control for Rehabilitation Robot Systems Using Actor-Critic Algorithm". Simulation and experiment as conducted on a rehabilitation robot demonstrate the effectiveness of the proposed control scheme.

UAVs, particularly small unmanned helicopters, are gaining more interest from worldwide researchers due to their hovering ability and vertical takeoff and landing (VTOL) capability. A. N. Khizer et al's "Stable Hovering Flight for a Small Unmanned Helicopter Using Fuzzy Control" proposes a Mamdani-based fuzzy control for small unmanned helicopter at hovering under turbulent environment. To show the effectiveness of the proposed fuzzy control method and its ability to cope with the external uncertainties, results are compared with a classical PD controller. Simulated results show that two-loop fuzzy controllers have a good ability to establish stable hovering for a class of unmanned rotorcraft in the presence of light turbulent environment.

Redundant actuators are usually adopted in safety-critical systems such as aircrafts, spacecrafts, and automotive vehicles, which require high-level of dependability. The goal of fault-tolerant control is to provide the feasible control input for a faulty system by reconfiguring a controller. I. Yang and
D. Lee's "Networked Fault-Tolerant Control Allocation for Multiple Actuator Failures" proposes an intelligent fault-tolerant control technique using network. Specifically, a faulttolerant control allocation method (FTCA) is proposed to tolerate failures on more than one actuator. The performance of the proposed FTCA method is verified by numerical simulations.

Motivated by the complex production management with difficulties in error-prone assembly system and inaccurate supply chain inventory, W. Xu et al.s "A Technical and Business Perspective on Wireless Sensor Network for Manufacturing Execution System" designs a manufacturing execution system (MES) architecture based on wireless sensor network (WSN) and proposes a key mutual inductance technical system and implementation architecture of MES based on sensor technology. The results of this paper have contributed to making the price and ordering decision on whether RFID should be adopted among members of the supply chain.

We hope that the readers will find the special issue interesting and stimulating and expect that the involved papers contribute to the further advance in the domain of intelligent monitoring and control. Moreover, we would like to express our heartfelt thanks to all the authors who submitted their papers and all the reviewers who helped in improving the papers for this special issue. 


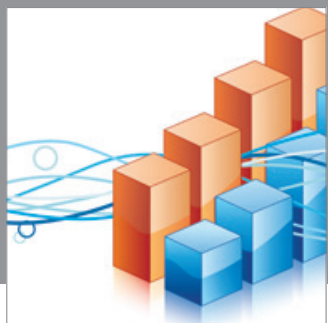

Advances in

Operations Research

mansans

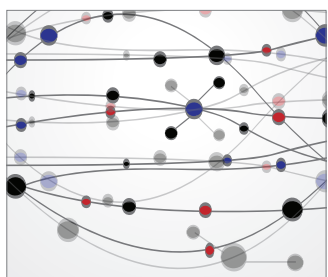

The Scientific World Journal
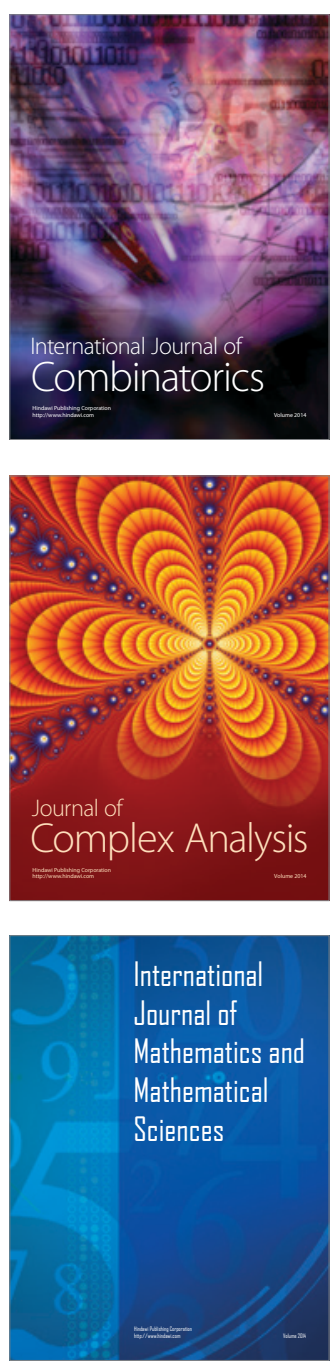
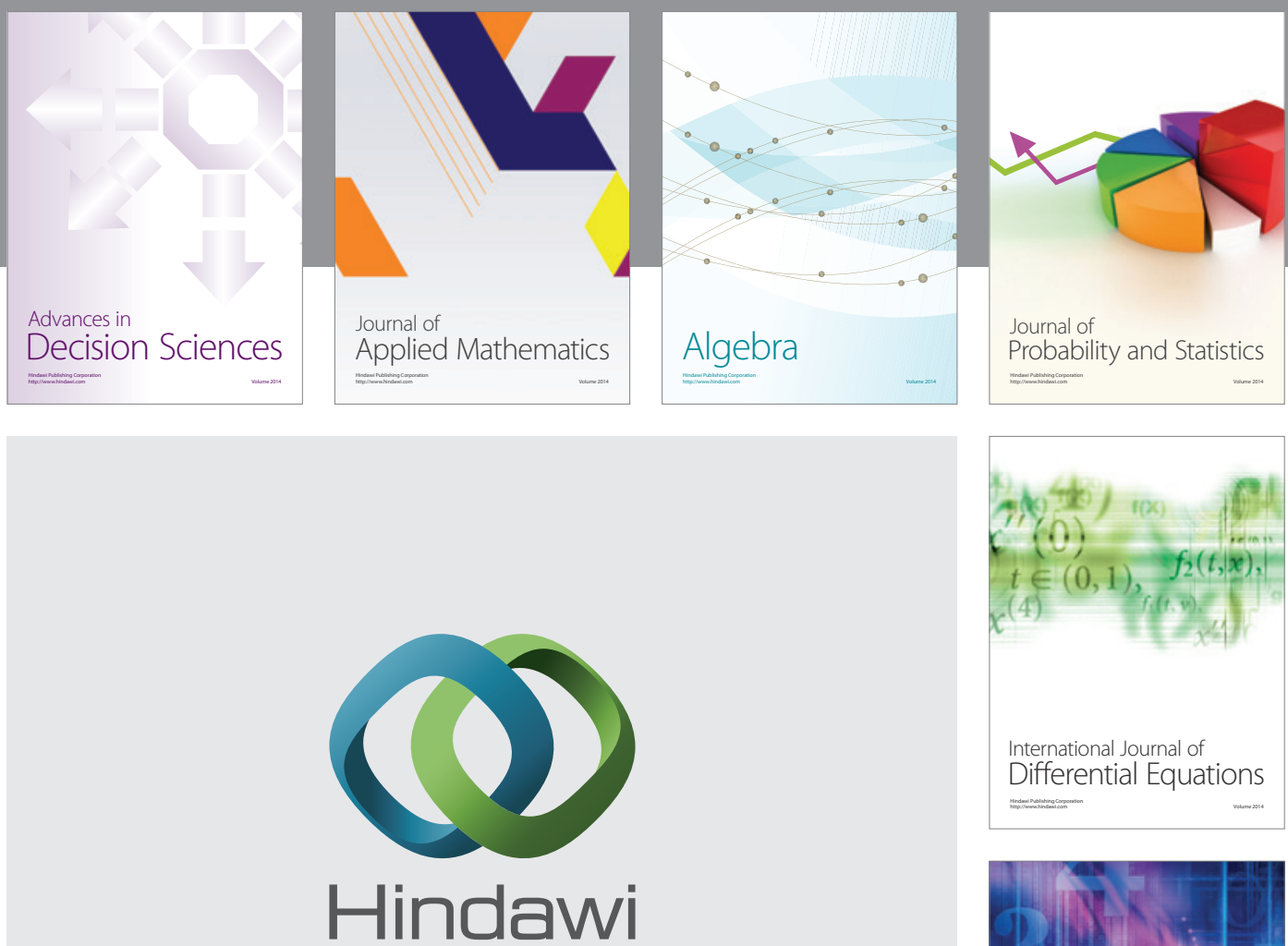

Submit your manuscripts at http://www.hindawi.com
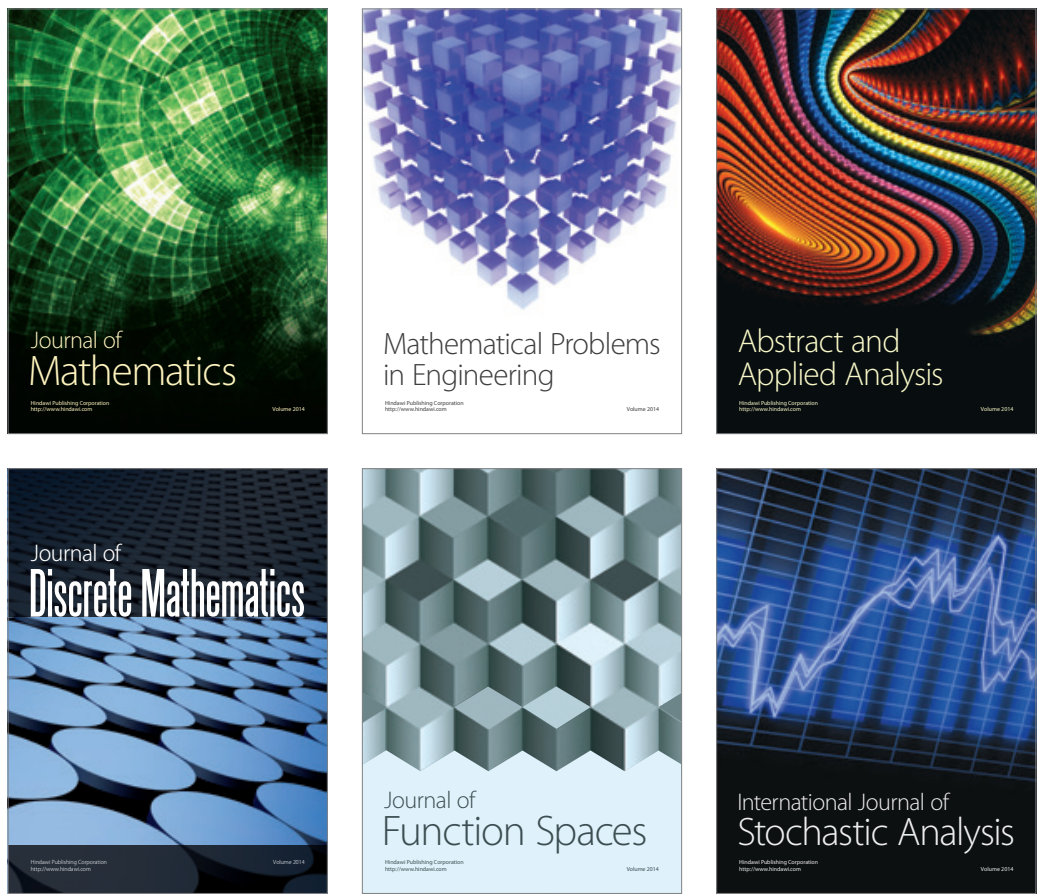

Journal of

Function Spaces

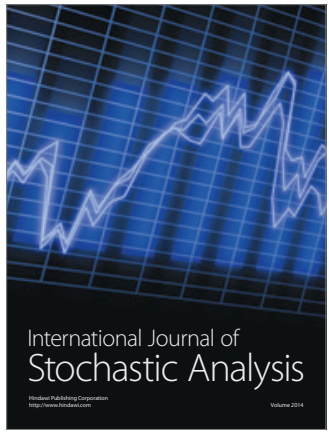

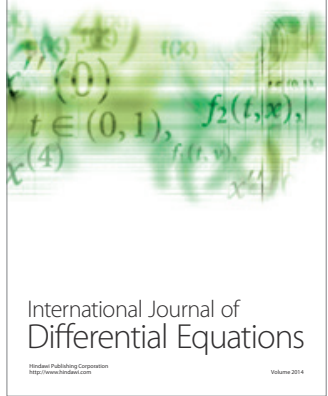
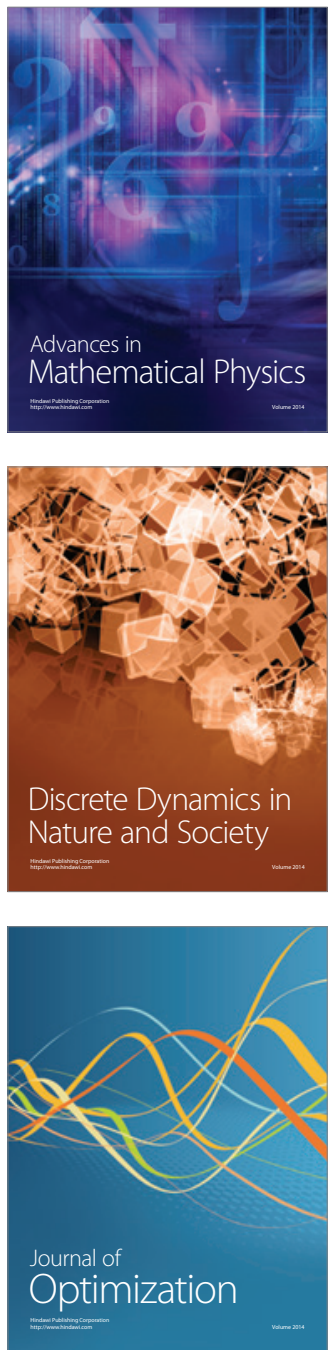